\title{
Front Matter: Volume 9699
}

, "Front Matter: Volume 9699," Proc. SPIE 9699, Optics and Biophotonics in Low-Resource Settings II, 969901 (13 May 2016); doi: 10.1117/12.2229253

SPIE. Event: SPIE BiOS, 2016, San Francisco, California, United States 


\section{PROGRESS IN BIOMEDICAL OPTICS AND IMAGING}

\section{Optics and Biophotonics in \\ Low-Resource Settings II}

David Levitz

Aydogan Ozcan

David Erickson

Editors

13-14 February 2016

San Francisco, California, United States

Sponsored and Published by

SPIE 
The papers in this volume were part of the technical conference cited on the cover and title page. Papers were selected and subject to review by the editors and conference program committee. Some conference presentations may not be available for publication. Additional papers and presentation recordings may be available online in the SPIE Digital Library at SPIEDigitallibrary.org.

The papers reflect the work and thoughts of the authors and are published herein as submitted. The publisher is not responsible for the validity of the information or for any outcomes resulting from reliance thereon.

Please use the following format to cite material from these proceedings:

Author(s), "Title of Paper," in Optics and Biophotonics in Low-Resource Settings II, edited by David Levitz, Aydogan Ozcan, David Erickson, Proceedings of SPIE Vol. 9699 (SPIE, Bellingham, WA, 2016) Six-digit Article CID Number.

ISSN: 1605-7422

ISSN: 2410-9045 (electronic)

ISBN: 9781628419337

Published by

SPIE

P.O. Box 10, Bellingham, Washington 98227-0010 USA

Telephone +1 3606763290 (Pacific Time) · Fax +1 3606471445

SPIE.org

Copyright @ 2016 , Society of Photo-Optical Instrumentation Engineers.

Copying of material in this book for internal or personal use, or for the internal or personal use of specific clients, beyond the fair use provisions granted by the U.S. Copyright Law is authorized by SPIE subject to payment of copying fees. The Transactional Reporting Service base fee for this volume is $\$ 18.00$ per article (or portion thereof), which should be paid directly to the Copyright Clearance Center (CCC), 222 Rosewood Drive, Danvers, MA 01923. Payment may also be made electronically through CCC Online at copyright.com. Other copying for republication, resale, advertising or promotion, or any form of systematic or multiple reproduction of any material in this book is prohibited except with permission in writing from the publisher. The CCC fee code is 1605$7422 / 16 / \$ 18.00$.

Printed in the United States of America.

Publication of record for individual papers is online in the SPIE Digital Library.

\section{SPIE. DIGITAL}

Paper Numbering: Proceedings of SPIE follow an e-First publication model. A unique citation identifier (CID) number is assigned to each article at the time of publication. Utilization of CIDs allows articles to be fully citable as soon as they are published online, and connects the same identifier to all online and print versions of the publication. SPIE uses a six-digit CID article numbering system structured as follows:

- The first four digits correspond to the SPIE volume number.

- The last two digits indicate publication order within the volume using a Base 36 numbering system employing both numerals and letters. These two-number sets start with $00,01,02,03,04$, $05,06,07,08,09,0 A, 0 B \ldots$ OZ, followed by 10-1Z, 20-2Z, etc. The CID Number appears on each page of the manuscript. 


\title{
Contents
}

\author{
$\checkmark$ Authors \\ vii Conference Committee
}

\section{LAB-ON-A-CHIP METHODS}

969902 Digital detection of biomarkers for high-sensitivity diagnostics at low-cost (Invited Paper) [9699-1]

969903 Single DNA imaging and length quantification through a mobile phone microscope [9699-2]

\section{FABRICATION AND 3D PRINTING IN OPTICAL SYSTEMS}

969906 Low cost flatbed scanner label-free biosensor [9699-5]

969907 Development and bench testing of a multi-spectral imaging technology built on a smartphone platform [9699-6]

969908 Mechanical and optical behavior of a tunable liquid lens using a variable cross section membrane: modeling results [9699-7]

969909 Development of a miniature multiple reference optical coherence tomography imaging device [9699-8]

9699 OA Wavelength scanning achieves pixel super-resolution in holographic on-chip microscopy [9699-9]

\section{FLUORESCENCE AND POLARIZATION METHODS}

9699 OE Evaluation of a polarization sensitive multiple reference optical coherence tomography system [9699-13]

\section{TRANSLATIONAL RESEARCH I: MICROSCOPY PLUS WHITE LIGHT IMAGING}

9699 0l Mobile colposcopy in urban and underserved suburban areas in Baja, California [9699-22]

9699 OL Custom field-of-view optofluidic imaging by synthetic digital holography [9699-25] 
9699 ON Thumb-size ultrasonic-assisted spectroscopic imager for in-situ glucose monitoring as optional sensor of conventional dialyzers [9699-16]

9699 OP The impact of relative intensity noise on the signal in multiple reference optical coherence tomography [9699-18]

$96990 Q \quad$ Flat lensless phase imager [9699-19]

TRANSLATIONAL RESEARCH II: NEW TECHNOLOGIES AND IMPLEMENTATIONS

9699 OR Initial clinical testing of a multi-spectral imaging system built on a smartphone platform [9699-26]

9699 OS Quantitative wound healing studies using a portable, low cost, handheld near-infrared optical scanner: preliminary sensitivity and specificity analysis [9699-27]

9699 OT A game-based crowdsourcing platform for rapidly training middle and high school students to perform biomedical image analysis [9699-28]

\section{POSTER SESSION}

9699 OV Melanoma detection using a mobile phone app [9699-30]

9699 OW Shed a light of wireless technology on portable mobile design of NIRS [9699-31]

9699 OY Potential applications of near infrared auto-fluorescence spectral polarized imaging for assessment of food quality [9699-33]

$96990 Z$ Evaluation of PpIX formation in Cervical Intraepithelial Neoplasia I (CIN) using widefield fluorescence images [9699-21] 


\section{Authors}

Numbers in the index correspond to the last two digits of the six-digit citation identifier (CID) article numbering system used in Proceedings of SPIE. The first four digits reflect the volume number. Base 36 numbering is employed for the last two digits and indicates the order of articles within the volume. Numbers start with 00, 01, 02, 03, 04, 05, 06, 07, 08, 09, 0A, 0B...0Z, followed by 10-1Z, 20-2Z, etc.

\begin{tabular}{|c|c|}
\hline Alexandrov, Sergey, OP & Luo, Wei, 03, OA \\
\hline Avci, Oguzhan, 02, 06 & Madiedo, Marta, Ol \\
\hline Aygun, Ugur, 06 & Marchesano, V., OL \\
\hline Bagnato, Vanderlei Salvador, $\mathrm{OZ}$ & McNamara, Paul M., 09, OE \\
\hline Bianco, V., OL & Mejia, Crystal, 03 \\
\hline Bolton, Frank J., 07, OR & Mink, Jonah W., OR \\
\hline Calixto, Sergio, 08 & Mori, Keita, ON \\
\hline Carbinatto, Fernanda M., $\mathrm{OZ}$ & Moser, Christophe, $0 Q$ \\
\hline Chan, Raymond Yan Lok, 03 & Neuhaus, Kai, OE, OP \\
\hline Chen, Jun, OY & Nishiyama, Akira, ON \\
\hline Chiang, Samuel, 03 & Nogo, Kosuke, ON \\
\hline Collins, Seán, 09 & O'Brien, Peter, 09 \\
\hline Connor, John, 02 & O'Gorman, Sean, OE \\
\hline Contreras, Sonia, ol & O'Riordan, Colm, 09 \\
\hline da Silva, Eduardo Venerando, $0 Z$ & Ozcan, Aydogan, 03, 0A, 0T \\
\hline Daaboul, George G., 02 & Ozkan, Haydar, 03 \\
\hline Dsouza, Roshan, OE, OP & Ozkumur, Ayca Yalcin, 06 \\
\hline E. Diniz, Luciano, OV & Paturzo, M., OL \\
\hline Ennser, K., OV & Perez-Clavijo, Francesco, OS \\
\hline Feizi, Alborz, OA & Pratavieira, Sebastião, $0 Z$ \\
\hline Feng, Steve, 03, От & Qi, Hangfei, 03 \\
\hline Ferraro, P., OL & Qi, Wei, ON \\
\hline Flores-Bustamante, Mario Carlos, 08 & Rodriguez, Suset, OS \\
\hline Fortunato, Thereza C., OZ & Rose, Donny, 07 \\
\hline Godavarty, Anuradha, OS & Rosete-Aguilar, Martha, 08 \\
\hline Gonzalez, Stephanie, OS & Rostykus, Manon, $0 Q$ \\
\hline Göröcs, Zoltan, OA & Safir, Amit, 07, 0l \\
\hline Greenbaum, Alon, OA & Scherr, Steve, 02 \\
\hline Hogan, Josh, 09, OE, OP & Sevenler, Derin D., 02, 06 \\
\hline Hosono, Satsuki, ON & Seymour, Elif Ç., 02,06 \\
\hline Hummel, Charles, OR & Shabbir, Faizan, 03 \\
\hline Huyet, Guillaume, OP & Shao, Lei, ОT \\
\hline Inada, Natalia Mayumi, OZ & Slepneva, Svetlana, OP \\
\hline Ishimaru, Ichiro, ON & Solis, Elizabeth, OS \\
\hline Jayachandran, Maanasa, OS & Subhash, Hrebesh, OP \\
\hline Kahn, Bruce S., Ol, OR & Sun, Yunlong, OW \\
\hline Kappel, Tara, 03 & Trueb, Jacob, 02 \\
\hline Kass, Alex J., 07 & Tseng, Derek, 03 \\
\hline Kawashima, Natsumi, ON & Ünlü, M. Selim, 02, 06 \\
\hline Ki, Sojung, OT & Urey, Hakan, 06 \\
\hline Kim, Eunso, OT & Villalobos, Octavio, ol \\
\hline Kim, Hannah, OT & Vollet Filho, José Dirceu, $\mathrm{OZ}$ \\
\hline Kurachi, Cristina, $\mathrm{OZ}$ & Wada, Kenji, ON \\
\hline Leahy, Martin J., 09, OE, OP & Wei, Qingshan, 03 \\
\hline Lei, Jiali, OS & Weiser, Reuven, 07 \\
\hline Levitz, David, 07, OI, OR & Wexler, Shraga, OR \\
\hline $\mathrm{Li}$, Ting, OW & Wigley, Stephen, OS \\
\hline Lombardi, Welington, $\mathrm{OZ}$ & Wilson, Carol, 09, OE, OP \\
\hline Lortlar Ünlü, Nese, 02 & Woo, Min-jae, OT \\
\hline
\end{tabular}


Yan, Eddie, 03

Zhang, Yibo, $0 A$

Zhou, Kenneth J., OY

Proc. of SPIE Vol. $9699969901-6$

Downloaded From: https://www.spiedigitallibrary.org/conference-proceedings-of-spie on 25 Apr 2023 Terms of Use: https://www.spiedigitallibrary.org/terms-of-use 


\title{
Conference Committee
}

\author{
Symposium Chairs
}

James G. Fujimoto, Massachusetts Institute of Technology

(United States)

R. Rox Anderson, Wellman Center for Photomedicine, Massachusetts General Hospital (United States) and Harvard School of Medicine (United States)

Program Track Chairs

Tuan Vo-Dinh, Fitzpatrick Institute for Photonics, Duke University (United States)

Anita Mahadevan-Jansen, Vanderbilt University (United States)

Conference Chairs

David Levitz, MobileODT (Israel)

Aydogan Ozcan, University of California, Los Angeles (United States)

David Erickson, Cornell University (United States)

Conference Program Committee

Gerard L. Coté, Texas A\&M University (United States)

Wolfgang Drexler, Medizinische Universität Wien (Austria)

Frances S. Ligler, North Carolina State University (United States)

Anita Mahadevan-Jansen, Vanderbilt University (United States)

Chetan A. Patil, Vanderbilt University (United States)

Nirmala Ramanujam, Duke University (United States)

Avi Rasooly, National Institutes of Health (United States)

Eric A. Swanson, OCT News (United States)

Sebastian Wachsmann-Hogiu, NSF Center for Biophotonics Science and Technology (United States)

Ian M. White, University of Maryland, College Park (United States)

\section{Session Chairs}

Lab-On-a-Chip Methods

David Levitz, MobileODT Ltd. (Israel)

Fabrication and 3D Printing in Optical Systems

Aydogan Ozcan, University of California, Los Angeles (United States) 
Fluorescence and Polarization Methods

David Erickson, Cornell University (United States)

Translational Research I: Microscopy Plus White Light Imaging

Anita Mahadevan-Jansen, Vanderbilt University (United States)

Blood Diagnostics

David Levitz, MobileODT Limited (Israel)

Translational Research II: New Technologies and Implementations

Euan McLeod, The University of Arizona (United States) 